\title{
Evaluation of Ultrafast Wave-Controlled Aliasing in Parallel Imaging 3D-FLAIR in the Visualization and Volumetric Estimation of Cerebral White Matter Lesions
}

\author{
DC. Ngamsombat, (D) A.L.M. Gonçalves Filho, (D) M.G.F. Longo, (D).F. Cauley, (D) K. Setsompop, (D).E. Kirsch, (DQ. Tian, (D). Fan, \\ (DD. Polak, (DW. Liu, (D)W.-C. Lo, (D) R. Gilberto González, (DP.W. Schaefer, (D) O. Rapalino, DJ. Conklin, and (D) S.Y. Huang
}

\begin{abstract}
BACKGROUND AND PURPOSE: Our aim was to evaluate an ultrafast 3D-FLAIR sequence using Wave-controlled aliasing in parallel imaging encoding (Wave-FLAIR) compared with standard 3D-FLAIR in the visualization and volumetric estimation of cerebral white matter lesions in a clinical setting.
\end{abstract}

MATERIALS AND METHODS: Forty-two consecutive patients underwent 3T brain MR imaging, including standard 3D-FLAIR (acceleration factor $=2$, scan time $=7$ minutes 50 seconds) and resolution-matched ultrafast Wave-FLAIR sequences (acceleration factor $=6$, scan time $=2$ minutes 45 seconds for the 20-channel coil; acceleration factor $=9$, scan time $=1$ minute 50 seconds for the 32-channel coil) as part of clinical evaluation for demyelinating disease. Automated segmentation of cerebral white matter lesions was performed using the Lesion Segmentation Tool in SPM. Student $t$ tests, intraclass correlation coefficients, relative lesion volume difference, and Dice similarity coefficients were used to compare volumetric measurements among sequences. Two blinded neuroradiologists evaluated the visualization of white matter lesions, artifacts, and overall diagnostic quality using a predefined 5point scale.

RESULTS: Standard and Wave-FLAIR sequences showed excellent agreement of lesion volumes with an intraclass correlation coefficient of 0.99 and mean Dice similarity coefficient of 0.97 (SD, 0.05) (range, 0.84-0.99). Wave-FLAIR was noninferior to standard FLAIR for visualization of lesions and motion. The diagnostic quality for Wave-FLAIR was slightly greater than for standard FLAIR for infratentorial lesions $(P<.001)$, and there were fewer pulsation artifacts on Wave-FLAIR compared with standard FLAIR $(P<.001)$.

CONCLUSIONS: Ultrafast Wave-FLAIR provides superior visualization of infratentorial lesions while preserving overall diagnostic quality and yields white matter lesion volumes comparable with those estimated using standard FLAIR. The availability of ultrafast Wave-FLAIR may facilitate the greater use of 3D-FLAIR sequences in the evaluation of patients with suspected demyelinating disease.

ABBREVIATIONS: CAIPI = controlled aliasing in parallel imaging; DSC $=$ Dice similarity coefficient; ICC $=$ intraclass correlation coefficient; LVD $=$ lesion volume difference; $M S=$ multiple sclerosis; $R=$ acceleration factor; $T A=$ scan time

W hite matter lesions secondary to demyelination in multiple sclerosis (MS) and related disorders typically present with high T2 signal and are best evaluated with FLAIR imaging, the standard sequence for cerebral white matter lesion detection. FLAIR

Received January 6, 2021; accepted after revision March 29.

From the Department of Radiology (C.N., A.L.M.G.F., M.G.F.L., S.F.C., K.S., J.E.K. Q.T., Q.F., R.G.G., P.W.S., O.R., J.C., S.Y.H.) and Athinoula A. Martinos Center for Biomedical Imaging (C.N., A.L.M.G.F., M.G.F.L., S.F.C., K.S., J.E.K., Q.T., Q.F., D.P., J.C., S.Y.H.), Massachusetts General Hospital, Boston, Massachusetts; Department of Radiology (C.N.), Faculty of Medicine, Siriraj Hospital, Mahidol University, Thailand; Harvard Medical School (A.L.M.G.F., M.G.F.L., S.F.C., K.S., J.E.K., Q.T., Q.F., R.G.G., P.W.S., O.R., J.C., S.Y.H.), Boston, Massachusetts; Harvard-MIT Division of Health Sciences and Technology (K.S., S.Y.H.), Massachusetts Institute of Technology, Cambridge, Massachusetts; Department of Physics and Astronomy (D.P.), Heidelberg University, Heidelberg, Germany; Siemens Healthcare GmbH (D.P., W.-C.L.), Erlangen, Germany; and Siemens Shenzhen Magnetic Resonance Ltd (W.L.), Shenzhen, China.

This work was supported by the National Institutes of Health, grant Nos: P41EB030006, K23-NS096056, R01-NS118187; Siemens (research support); and Massachusetts General Hospital Claflin Distinguished Scholar Award. is a T2-weighted sequence with nulling of the CSF signal, which increases the contrast between lesions and CSF/cerebral sulci and ventricles and improves white matter lesion detection and analysis. ${ }^{1}$

Quantification of cerebral white matter lesion volume has become increasingly feasible for routine clinical evaluation and use in clinical trials of MS therapies due to the availability of automated segmentation tools and 3D fast spin-echo FLAIR sequences, which delineate cerebral white matter lesions at high isotropic resolution. The Lesion Segmentation Tool (LST; https://www.applied-statistics.

Please address correspondence to Susie Y. Huang, MD, PhD, Division of Neuroradiology, Department of Radiology, Massachusetts General Hospital, 55 Fruit St, GRB-273A, Boston, MA, 02114; e-mail: susie.huang@mgh.harvard.edu

- Indicates open access to non-subscribers at www.ajnr.org

Indicates article with online supplemental data.

http://dx.doi.org/10.3174/ajnr.A7191 
de/lst.html), a promising tool for automated segmentation of T2hyperintense lesions on FLAIR images, was developed for the quantification of MS lesion volumes and has been shown to have good agreement with manual segmentation by expert reviewers. ${ }^{2-7}$ However, the high-resolution 3D-FLAIR images required as input for this tool have long acquisition times, limiting the widespread use of automated lesion segmentation in clinical practice.

Wave-controlled aliasing in parallel imaging (CAIPI) is a recently developed fast acquisition technology that synergistically combines and extends 2 controlled aliasing approaches, 2DCAIPI and bunch phase encoding, ${ }^{8}$ to achieve controlled aliasing in all 3 spatial directions $(x, y, z)$. By taking full advantage of the $3 \mathrm{D}$ coil sensitivity information, Wave-CAIPI offers high acceleration factors with negligible artifacts and g-factor penalty. ${ }^{9,10} 3 \mathrm{D}$ FLAIR acquired with Wave-CAIPI cuts the scan time down by more than half, possibly facilitating the broader clinical application of 3D-FLAIR in the evaluation of white matter diseases such as MS.

The goal of this study was to evaluate an ultrafast WaveCAIPI 3D-FLAIR sequence (Wave-FLAIR) ${ }^{11,12}$ acquired in less than half the time of standard 3D-FLAIR for quantitative and qualitative analyses of cerebral white matter lesions.

\section{MATERIALS AND METHODS Subjects and Study Design}

This study was approved by the institutional review board of Partners Healthcare and was Health Insurance Portability and Accountability Act-compliant. A prospective comparative study was performed at a single institution from April 2019 to March 2020. Forty-two consecutive patients undergoing brain MR imaging as part of routine clinical work-up and/or surveillance for MS and other white matter diseases were enrolled.

\section{Data Acquisition}

MR imaging was performed on 1 of 2 clinical 3T MR imaging scanners (Magnetom Prisma; Siemens) using 20- or 32-channel multiarray receiver coils, depending on the fit and comfort of the patient. Each scan included a standard 3D sampling perfection with application optimized contrasts using different flip angle evolutions (SPACE sequence; Siemens) FLAIR sequence (acceleration factor $[R]=2$, scan time $[\mathrm{TA}]=7$ minutes 15 seconds), and resolution-matched ultrafast $3 \mathrm{D}$ Wave SPACE-FLAIR $(\mathrm{R}=6, \mathrm{TA}=7$ minutes 45 seconds for the 20-channel coil and $\mathrm{R}=9$, $\mathrm{TA}=1$ minute 50 seconds for the 32 -channel coil) sequences. The order of the Wave and standard FLAIR sequences was reversed half-way through the study period to minimize any potential bias due to the order of acquisition. Detailed acquisition parameters for the standard and Wave SPACE-FLAIR sequences are shown in the Table.

\section{White Matter Lesion Analysis}

Quantitative Analysis. Cerebral white matter lesions were segmented using the lesion prediction algorithm implemented in the LST toolbox, Version 2.0.15 in SPM (http://www.fil.ion.ucl.ac.uk/ $\mathrm{spm} /$ software/spm12). ${ }^{2}$ Lesion probability maps generated by the lesion prediction algorithm from the standard and Wave-FLAIR sequences were compared using the longitudinal pipeline in LST.
Acquisition parameters for standard and wave SPACE FLAIR sequences

\begin{tabular}{lcc}
\hline \multicolumn{1}{c}{ Parameters } & Standard & Wave SPACE FLAIR \\
\hline FOV read (mm) & $256 \times 256$ & $256 \times 256$ \\
FOV phase (\%) & 100 & 100 \\
Matrix size & $256 \times 256$ & $256 \times 256$ \\
$\begin{array}{l}\text { Section thickness } \\
(\mathrm{mm})\end{array}$ & 1 & 1 \\
TR/TE/TI (ms) & $5000 / 390 / 1800$ & $5000 / 392 / 1800$ \\
Acceleration factor & & \\
20-channel & 2 & 6 \\
32-channel & 2 & 9 \\
Bandwidth (Hx/pixel) & 750 & 650 \\
Scan time (sec) & & 2 minutes 45 \\
20-channel & 7 minutes 15 & seconds \\
& seconds & 1 minute 50 \\
32-channel & 7 minutes 15 & seconds \\
\hline
\end{tabular}

Binarized lesion maps were created on the basis of the lesion probability maps derived from standard and Wave-FLAIR sequences using default threshold values set by the LST for all subjects. Lesions in each brain region, including periventricular, juxtacortical, infratentorial, deep white matter and subcortical white matter and deep gray matter regions, were identified and labeled by a neuroradiologist blinded to sequence type and order using the Island Tools Editor in 3D Slicer, Version 4.10.2 (https:// www.slicer.org/) for further analyses. Lesion volume and number were compared in each brain region between the standard and Wave-FLAIR images.

Qualitative Analysis. Two neuroradiologists (J.C. and A.L.M.G.F., with 8 years of experience each), blinded to sequence type. performed a head-to-head comparison of the images. A predefined 5 -point scale was used for grading white matter lesions in the locations specified in the McDonald criteria (ie, periventricular, juxtacortical, and infratentorial locations $)^{13}$ and other locations, including subcortical white matter, deep white matter, and deep gray matter. Other variables that were evaluated included motion, pulsation artifacts, noise, and overall diagnostic quality (Online Supplemental Data). All images were evaluated in a randomized and blinded fashion. A third reader adjudicated the discrepancies (S.Y.H., with 10 years of experience).

Statistical Analysis. All statistical calculations were performed in Matlab software, Version 9.4 (MathWorks) and R statistical and computing software, Version 3.4.3 (http://www.r-project.org/). The Student $t$ test was used to assess a statistically significant difference in lesion volumes and lesion numbers between the standard and Wave sequences. The correlations between standard and Wave-FLAIR lesion volumes and lesion numbers were assessed using Pearson correlation coefficients. The 2-way random intraclass correlation coefficient (ICC) was calculated to assess absolute agreement and consistency between standard and WaveFLAIR lesion volumes ${ }^{14}$ as a measure of volumetric precision, with a higher ICC signifying higher intermeasurement agreement. ${ }^{5,7,14,15}$ Relative lesion volume difference (LVD) ${ }^{16}$ was also used to compare standard and Wave-FLAIR lesion volumes, defined as LVD $=($ Total Lesion Volume on Wave - Total Lesion Volume on Standard) / Total Lesion Volume on Standard. LVD 
was used as a simple measure of the difference in volumes between standard- and Wave-FLAIR-segmented lesions as a fraction of the segmented lesion volume on standard FLAIR serving as the reference. To assess the overlap between segmented lesion voxels that might not be captured by LVD, we calculated the Dice similarity coefficient (DSC) as another indicator of the segmentation algorithm overlap between the 2 sequences. ${ }^{3,5,7}$ DSC is used in the literature to estimate regional spatial overlap, rather than just the agreement in volumetric values. ${ }^{17}$ The DSC provides information on the size and structure of an analyzed region; consequently, it is a more robust way to evaluate the similarity between 2 images. The Dice similarity coefficient of the standard and Wave images was expressed as Dice (Standard, Wave) $=2 \times$ $\mid$ Intersection (Standard, Wave) $\mid /(\mid$ Standard $|+|$ Wave $\mid)$. DSC measures have values between 0 and 1 , with higher values indicating better agreement. ${ }^{17}$

In the head-to-head qualitative analysis of the standard-versusWave-FLAIR images, we tested for noninferiority of Wave compared with standard FLAIR $^{18}$ using a noninferiority margin $(\Delta)$ of $15 \%$ as previously established. ${ }^{19}$ The null hypothesis $\left(\mathrm{H}_{0}\right)$ was that the proportion of cases in which standard FLAIR was preferred over Wave-FLAIR was $>15 \%$. We used the $z$ statistic to calculate the probability of the standard FLAIR being preferred over the Wave-FLAIR sequence in $>15 \%$ of cases $\left(\mathrm{H}_{0}>\Delta\right)$, with a type I error rate $(\alpha)$ of .05 . The required sample size was estimated as described $^{20}$ for a single proportion (the proportion of subjects in which visualization of lesions was preferred on standard over Wave-FLAIR). For a type I error rate $(\alpha)$ of .05 , a power $(1-\beta)$ of 0.90 , and noninferiority margin of $15 \%$, a minimum of 24 cases was required.

For all statistical analyses, corrections for multiple comparison were conducted on the basis of the false discovery rate adjustment, with a false discovery rate threshold of 0.05 . The raw uncorrected $P$ values surviving false discovery rate correction are reported here.

\section{RESULTS}

Forty-two adults participated in the comparative evaluation of the standard and Wave-FLAIR sequences. Demographic information on the study subjects, including age, sex, and clinical indication for undergoing MR imaging is shown in the Online Supplemental Data. A total of 38 patients of $42(90.5 \%)$ had white matter lesions. Thirty-six patients $(85.7 \%)$ were scanned with the 20-channel coil. Twenty-two patients were scanned with standard before Wave-FLAIR, while 20 were scanned with Wave before standard FLAIR.

In this section, we first present the quantitative comparison of lesion volumes followed by the qualitative evaluation of image quality. Standard and Wave-FLAIR images were evaluated in each brain region using predefined evaluation metrics described in the Materials and Methods section.

Six patients were excluded from the LST quantitative analysis because they had no detectable lesions or severe motion artifacts resulting in failure of the automated LST processing stream. On the whole-brain level, the standard and Wave-FLAIR sequences showed no significant difference in lesion volume (167,800 versus $168,130 \mathrm{~mm}^{3}, P=.99$ ) or lesion number (520 versus 529, $P=.91$ )

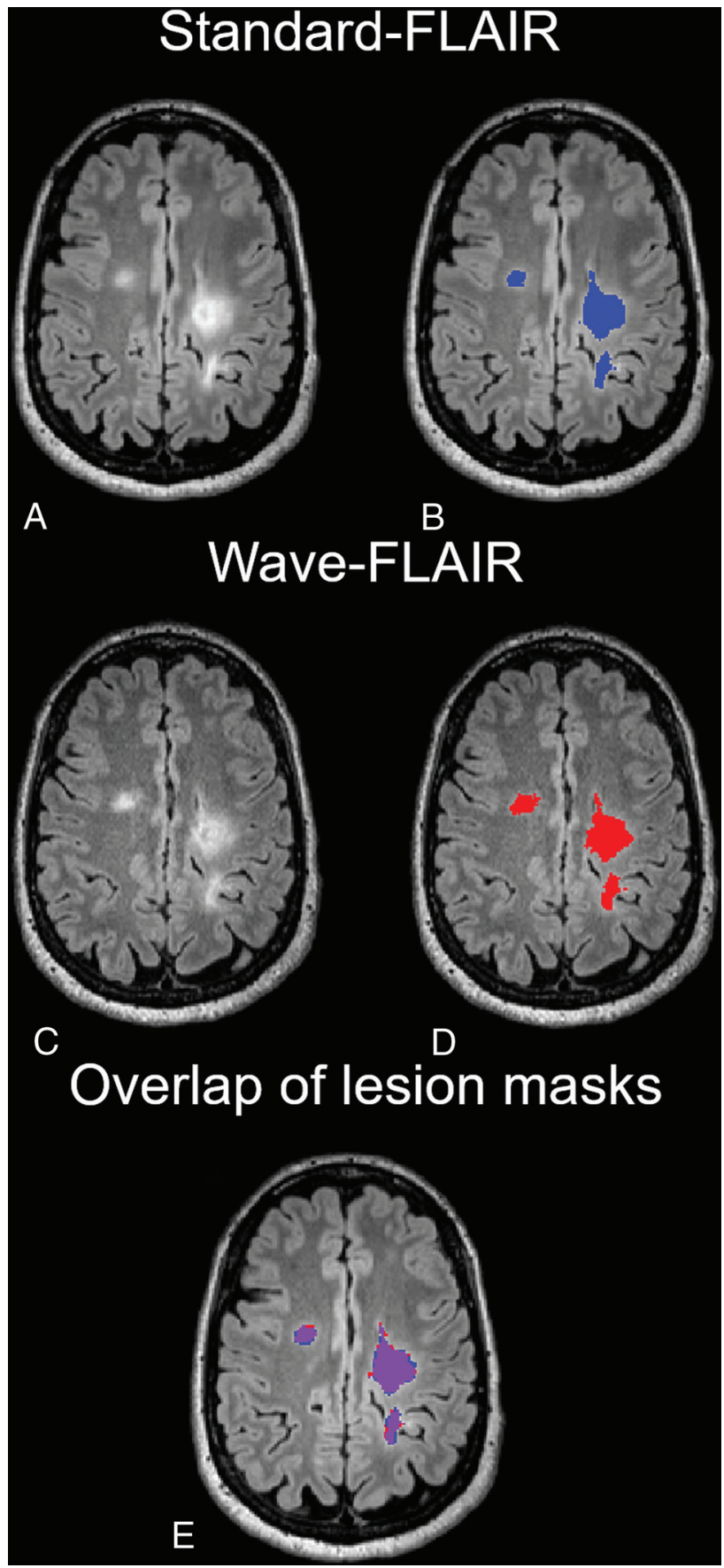

FIG 1. Comparison of standard and Wave-FLAIR images and lesion masks. $A$, Standard FLAIR image with the lesion mask overlaid ( $B$, blue). Wave-FLAIR image $(C)$ with the lesion mask $(D)$ overlaid (red). $E$, Lesion masks from standard and Wave-FLAIR images overlaid on the same image (purple indicates overlap of the lesion masks).

as segmented by LST. The ICC between standard and WaveFLAIR was 0.99. The relative LVD was 0.01 (SD, 0.05) (range, $-0.012-0.02$ ), and the DSC was 0.97 (SD, 0.05) (range, 0.840.99) (Online Supplemental Data). For lesions in each brain region (Figs 1 and 2), there was excellent correlation between standard and Wave-FLAIR images for lesion volume and lesion number (Online Supplemental Data), with no significant difference in lesion volumes $(P>.98)$ (Online Supplemental Data) or 


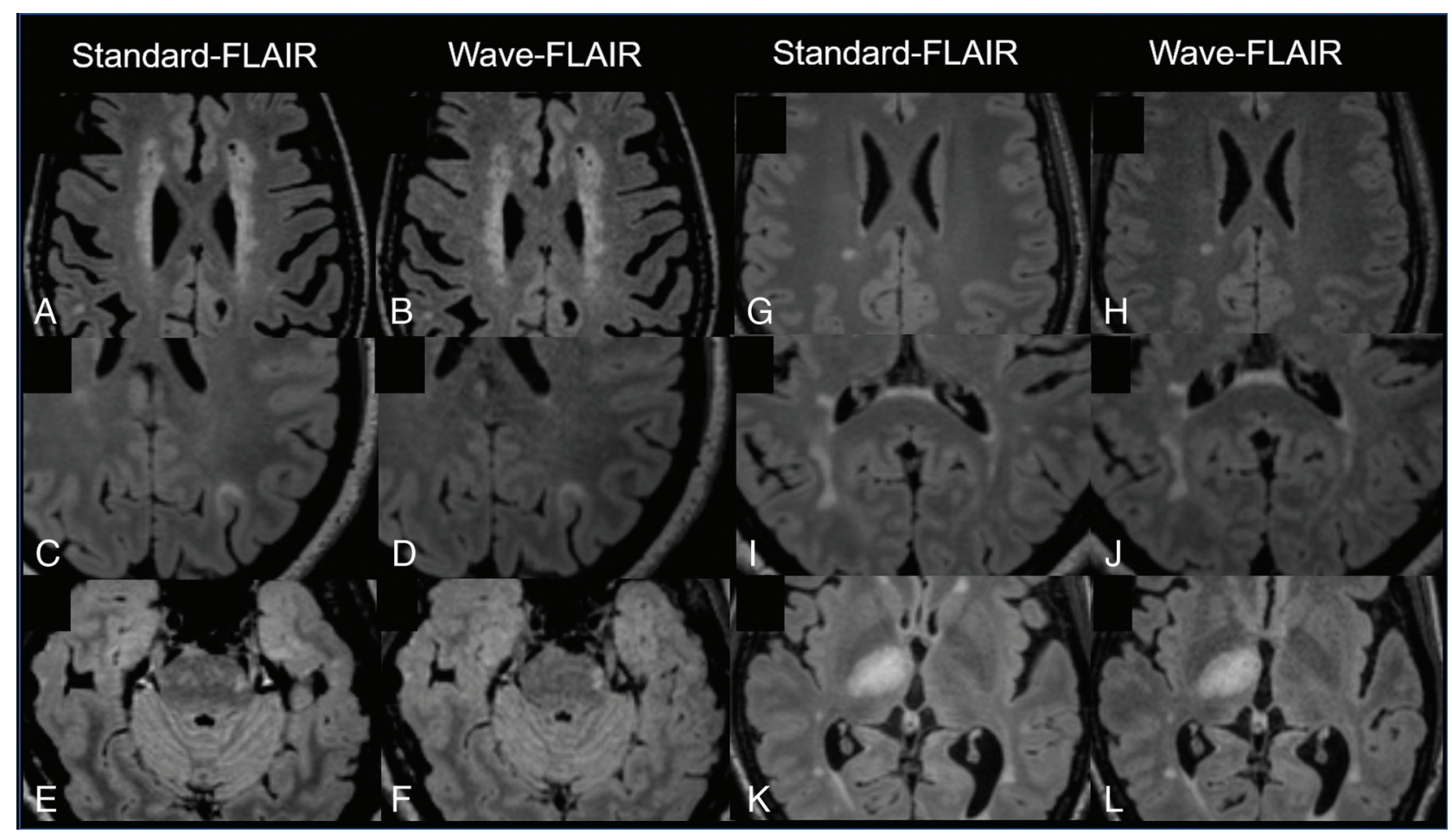

FIG 2. Comparison of MS lesions in different brain regions on standard and Wave-FLAIR images. The standard images $(A, C, E, G, I, K)$ are on the left, and the Wave-FLAIR images $(B, D, F, H, J, L)$ are on the right of each image pair. Lesion locations include the periventricular $(A$ and $B)$, juxtacortical $(C$ and $D)$, infratentorial $(E$ and $F)$, and deep white matter $(G$ and $H)$ and subcortical white matter $(I$ and $/)$ and deep gray matter $(K$ and $L)$.

lesion numbers $(P>.89)$ (Online Supplemental Data) as segmented by LST for each sequence. The LVDs were very low for all brain regions $(<2 \%)$, and the Dice coefficient was $>0.9$ between the 2 sequences for all brain regions, including the periventricular, deep, and subcortical white matter and deep gray matter, with the exception of the infratentorial white matter $(\mathrm{DSC}=0.84)$ (Online Supplemental Data).

Wave-FLAIR was equivalent to standard FLAIR for the visualization of lesions in the subcortical and deep white matter and deep gray matter $(P<.001)$ and was noninferior to standard FLAIR in the visualization of periventricular $(P<.001)$, juxtacortical $(P<.006)$, and infratentorial lesions $(P<.001)$ (Fig 3$)$. There was a slightly greater preference for Wave-FLAIR in the visualization of infratentorial lesions compared with the standard FLAIR sequence.

Wave-FLAIR was noninferior to standard FLAIR in terms of motion $(P<.001)$, with a slightly higher proportion of cases favoring Wave-FLAIR (7\%) compared with standard FLAIR (5\%) (Fig 3). Wave-FLAIR demonstrated fewer pulsation artifacts $(P<.001)$ in areas such as the brain stem (Fig $2 E)$. Wave-FLAIR demonstrated more noise overall but was ultimately noninferior in overall diagnostic quality compared with standard FLAIR $(P<.001)$ (Fig 3).

\section{DISCUSSION}

In this work, we performed a systematic quantitative evaluation of cerebral white matter lesion volumes and a qualitative evaluation of lesion conspicuity, artifacts, and overall diagnostic quality of an ultrafast Wave-SPACE FLAIR sequence, which was $>2.5$ times faster than the standard 3D SPACE FLAIR sequence. The results showed excellent agreement and spatial overlap between Wave- and standard FLAIR white matter lesion volumes estimated by the automated segmentation tool LST. Experienced neuroradiologists rated the accelerated Wave-FLAIR images as providing equivalent visualization of lesions in the supratentorial and infratentorial white matter compared with the standard FLAIR images, with preserved diagnostic quality. The findings support the broader application of ultrafast Wave FLAIR sequences in the evaluation of patients with white matter diseases.

White matter lesion quantification has become an increasingly important tool for characterizing the burden of disease in MS in both routine clinical evaluation and clinical trials. ${ }^{4}$ Manual white matter lesion segmentation is time-consuming and has the risk of rater bias. In addition, high image quality is needed for the best quantification. Automated lesion segmentation tools that require no or minimal training data are publicly available, including the LST, $^{3}$ LesionTOADS (https://github.com/sergivalverde/lesiontoads), ${ }^{16,21}$ SALEM Lesion Segmentation, ${ }^{22}$ and Automated Statistical Interference for Segmentation. ${ }^{23}$ We chose to use LST with the lesion probability algorithm (LST-lesion prediction algorithm $)^{2}$ because it has high accuracy in automatically segmenting MS lesions compared with manual segmentation ${ }^{7}$ and requires only FLAIR images as input. ${ }^{2,7}$ Here, we found comparable volumes and numbers of white matter lesions as segmented by LST on ultrafast Wave-FLAIR images compared with standard FLAIR, despite the slightly greater image noise observed in the WaveFLAIR images. These findings were supported by the high ICC (0.99) and, overall, a very small LVD in all brain regions $(<2 \%)$ 


\section{Head-to-Head Analysis, Standard vs. Wave-FLAIR}

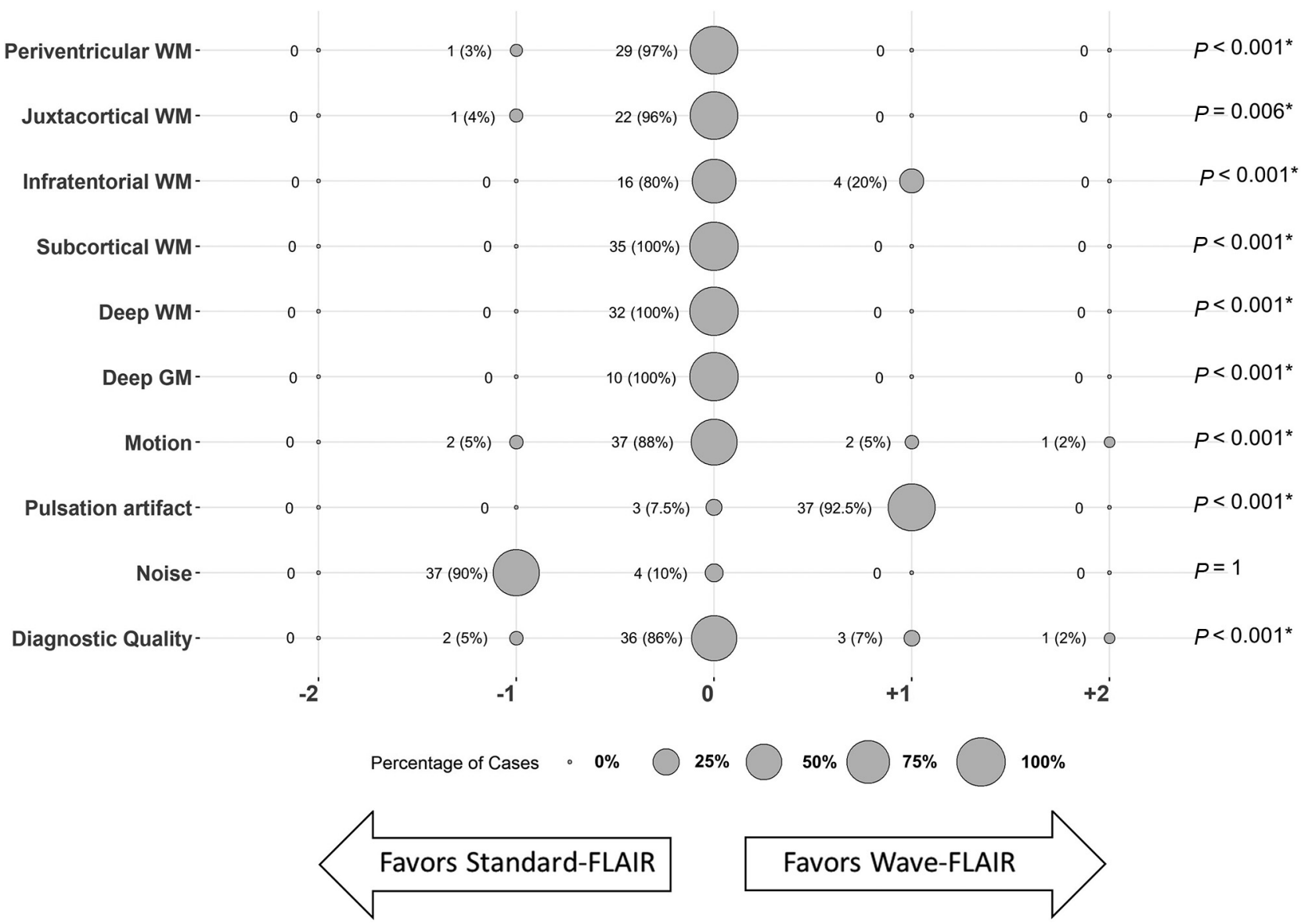

FIG 3. Balloon plot showing the head-to-head comparison of standard-versus-Wave-FLAIR images. The size of each balloon represents the relative percentage of cases with a given score; $P$ values for noninferiority testing are specified at the end of each row. The number of cases (percentage) are also noted adjacent to each balloon. The asterisk denotes significance following correction for multiple comparisons (false discovery rate threshold of 0.05 ).

between the 2 sequences. The DSC was $>0.9$ in all supratentorial regions and slightly lower for the infratentorial region $(\mathrm{DSC}=0.84)$. The lesser degree of agreement between the 2 sequences for infratentorial lesions likely reflects the known difficulty in detecting infratentorial lesions on FLAIR contrast images, which are, overall, less sensitive for posterior fossa lesions, ${ }^{24,25}$ resulting in a greater difference in voxels identified as part of lesions between the 2 sequences. If validated in larger studies, the overall excellent agreement in lesion quantification between Wave- and standard FLAIR suggests that Wave-FLAIR could potentially replace standard FLAIR for white matter lesion quantification in clinical and research studies using imaging in MS.

In addition to volumetric measures, we also included visual evaluation of the images by multiple neuroradiologists to assess the diagnostic performance of the Wave-FLAIR sequence, which is an important part of the patient's clinical evaluation. WaveFLAIR provided visualization of lesions in all locations, comparable with the standard sequence. The slightly greater image noise noted in the head-to-head comparison of the accelerated WaveFLAIR images compared with the standard FLAIR images (Fig 3) did not compromise the overall diagnostic quality, as illustrated in Fig 1 (see Fig $1 A,-C$ for representative standard-versus-Wave images). Furthermore, the Wave-FLAIR images showed reduced pulsatile flow artifacts in the posterior fossa, which contributed to improved visualization of infratentorial lesions, as illustrated in Fig $2 E$, $-F$. The standard FLAIR image (Fig 2) had more pulsation artifacts in the brainstem, which could lead to potential misinterpretation of a small T2-hyperintense lesion in the left lateral aspect of the pons as an artifact. This lesion clearly appeared as a distinct lesion in the Wave-FLAIR image (Fig $2 F$ ) without conspicuous artifacts in this area. The improved visualization of T2/ FLAIR hyperintense lesions in the brainstem and cerebellum indicates that Wave-FLAIR may be useful in the evaluation of not only white matter disease burden but also other conditions such as stroke and tumors.

The decreased scan time of Wave-FLAIR offers synergistic benefits for the comprehensive evaluation of white matter lesions in multiple sclerosis. Highly accelerated imaging with WaveCAIPI has been shown to reduce motion artifacts and improve the visualization of small lesions. ${ }^{19}$ In the current study, WaveFLAIR was noninferior to standard FLAIR for motion artifacts. 
One explanation for the less pronounced improvement in motion artifacts on Wave-FLAIR is that most patients (85.7\%) were scanned with the 20-channel coil, for which Wave-FLAIR was still 2:45 minutes in duration. We expect that motion artifacts would be further reduced if more patients were scanned using the 32channel coil (1:50-minute acquisition). The time-savings incurred by Wave-FLAIR may become more obvious when aggregated with other optimized fast $2 \mathrm{D}$ and $3 \mathrm{D}$ sequences. ${ }^{12,19,26,27}$ For example, at our institution, we have implemented the WaveFLAIR sequence along with optimized simultaneous multislice diffusion-weighted imaging, Wave T2 SPACE, Wave-SWI, and pre- and postcontrast Wave-T1 MPRAGE sequences in the clinical MS brain MR imaging protocol, bringing the total scan time for this protocol $<20$ minutes. The ability to acquire multiple 3D sequences with complementary contrasts efficiently, such as Wave-FLAIR and Wave-SWI, may encourage the greater adoption of promising imaging signs such as the central vein sign and paramagnetic rim sign, ${ }^{28-30}$ which have greater specificity for demyelinating lesions in MS and, in the case of the paramagnetic rim sign, may have prognostic value in identifying lesions with chronic active inflammation associated with greater disability. ${ }^{31}$ We envision that the systematic incorporation of highly accelerated 3D-Wave-CAIPI sequences into clinical MR imaging protocols will provide more information per unit of time and enable the more comprehensive evaluation of a wide range of neurologic disorders, thereby advancing clinical care and clinical research along multiple fronts.

Our study had some limitations. To reduce observer bias in the qualitative evaluation, the raters were blinded to the pulse sequence, but inevitably some imaging features could help to identify the sequence and introduce observer bias. In addition, image quality also depends on the order of acquisition for each pulse sequence. In general, images acquired later in the examination would be expected to have more motion. We sought to mitigate against this bias by randomizing the acquisition order of the sequences during the study. Finally, LST underestimated total lesion numbers in patients with a high lesion load, which decreased the agreement between Wave- and standard FLAIR in certain cases, such as the assessment of confluent lesions in the periventricular area.

\section{CONCLUSIONS}

Quantitative white matter lesion volumes and qualitative evaluation of white matter lesions imaged with an ultrafast, $<3$-minute, Wave-FLAIR sequence showed excellent agreement, with standard FLAIR images requiring more than double the scan time in patients undergoing clinical evaluation for demyelinating disease. The findings are derived from MR imaging examinations that were performed as part of a routine clinical work-up and/or surveillance for MS and reflect the performance of these sequences in a realistic clinical setting. The availability of ultrafast $3 \mathrm{D}$ sequences such as Wave-FLAIR may facilitate the more comprehensive evaluation of white matter lesions in MS and other white matter diseases.
Disclosures: Augusto Lio Goncalves Filho-UNRELATED: Employment: Massachusetts General Hospital. Stephen F. Cauley—RELATED: Grant: Siemens, Comments: Siemens research grant*; UNRELATED: Grants/Grants Pending: Siemens, Comments: Siemens research grant.* Kawin Setsompop-RELATED: Grant: National Institutes of Health, Comments: R01EB020613. John E. KirschUNRELATED: Grants/Grants Pending: National Institutes of Health grant. Qiyuan Tian-UNRELATED: Employment: Massachusetts General Hospital, Harvard Medical School. Daniel Polak-UNRELATED: Employment: Siemens. Wei LiuUNRELATED: Employment: Siemens. Wei-Ching Lo-UNRELATED: Employment: Siemens. Otto Rapalino-UNRELATED: Travel/Accommodations/Meeting Expenses Unrelated to Activities Listed: GE Healthcare. John ConklinUNRELATED: Grants/Grants Pending: Radiological Society of North America, Comments: Radiological Society of North America Research Seed Grant.* Susie Y. Huang-RELATED: Grant: Siemens, Comments: grant to support fast neuroimaging research.* ${ }^{*}$ Money paid to the institution.

\section{REFERENCES}

1. Filippi M, Yousry T, Baratti C, et al. Quantitative assessment of MRI lesion load in multiple sclerosis. a comparison of conventional spin-echo with fast fluid-attenuated inversion recovery. Brain 1996;119(Pt 4):1349-55 CrossRef Medline

2. Schmidt P. Bayesian inference for structured additive regression models for large-scale problems with applications to medical imaging. LMU München; 2017. https://edoc.ub.uni-muenchen.de/20373/. Accessed July 22, 2020

3. Schmidt P, Gaser C, Arsic M, et al. An automated tool for detection of FLAIR-hyperintense white-matter lesions in multiple sclerosis. Neuroimage 2012;59:3774-83 CrossRef Medline

4. Schmidt P, Pongratz V, Kuster P, et al. Automated segmentation of changes in FLAIR-hyperintense white matter lesions in multiple sclerosis on serial magnetic resonance imaging. Neuroimage Clin 2019;23:101849 CrossRef Medline

5. Weeda MM, Brouwer I, de Vos ML, et al. Comparing lesion segmentation methods in multiple sclerosis: Input from one manually delineated subject is sufficient for accurate lesion segmentation. Neuroimage Clin 2019;24:102074 CrossRef Medline

6. Valverde S, Oliver A, Roura E, et al. Quantifying brain tissue volume in multiple sclerosis with automated lesion segmentation and filling. Neuroimage Clin 2015;9:640-47 CrossRef Medline

7. Egger C, Opfer R, Wang C, et al. MRI FLAIR lesion segmentation in multiple sclerosis: does automated segmentation hold up with manual annotation? Neuroimage Clin 2017;13:264-70 CrossRef Medline

8. Moriguchi H, Duerk JL. Bunched phase encoding (BPE): a new fast data acquisition method in MRI. Magn Reson Med 2006;55:633-48 CrossRef Medline

9. Cauley SF, Setsompop K, Bilgic B, et al. Autocalibrated wave-CAIPI reconstruction; Joint optimization of $\mathbf{k}$-space trajectory and parallel imaging reconstruction. Magn Reson Med 2017;78:1093-99 CrossRef Medline

10. Bilgic B, Gagoski BA, Cauley SF, et al. Wave-CAIPI for highly accelerated 3D imaging. Magn Reson Med 2015;73:2152-62 CrossRef Medline

11. Polak D, Setsompop K, Cauley SF, et al. Wave-CAIPI for highly accelerated MP-RAGE imaging. Magn Reson Med 2018;79:401-06 CrossRef Medline

12. Polak D, Cauley S, Huang SY, et al. Highly-accelerated volumetric brain examination using optimized wave-CAIPI encoding. J Magn Reson Imaging 2019;50:961-74 CrossRef Medline

13. Filippi M, Preziosa P, Banwell BL, et al. Assessment of lesions on magnetic resonance imaging in multiple sclerosis: practical guidelines. Brain 2019;142:1858-75 CrossRef Medline

14. Reuter M, Schmansky NJ, Rosas HD, et al. Within-subject template estimation for unbiased longitudinal image analysis. Neuroimage 2012;61:1402-18 CrossRef Medline 
15. Jain S, Sima DM, Ribbens A, et al. Automatic segmentation and volumetry of multiple sclerosis brain lesions from MR images. Neuroimage Clin 2015;8:367-75 CrossRef Medline

16. Le M, Tang LY, Hernandez-Torres E, et al. FLAIR(2) improves LesionTOADS automatic segmentation of multiple sclerosis lesions in non-homogenized, multi-center, 2D clinical magnetic resonance images. Neuroimage Clin 2019;23:101918 CrossRef Medline

17. Dice LR. Measures of the amount of ecological association between species. Ecology 1945;26:297-302

18. Ahn S, Park SH, Lee KH. How to demonstrate similarity by using noninferiority and equivalence statistical testing in radiology research. Radiology 2013;267:328-38 CrossRef Medline

19. Conklin J, Longo MG, Cauley SF, et al. Validation of highly accelerated wave-CAIPI SWI compared with conventional SWI and T2*weighted gradient recalled-echo for routine clinical brain MRI at 3T. AJNR Am J Neuroradiol 2019;40:2073-80 CrossRef Medline

20. Chow SS, Shao J. Wang H. Sample Size Calculation in Clinical Research. Marcel Dekker; 2003

21. Shiee N, Bazin PL, Ozturk A, et al. A topology-preserving approach to the segmentation of brain images with multiple sclerosis lesions. Neuroimage 2010;49:1524-35 CrossRef Medline

22. Roura E, Oliver A, Cabezas M, et al. A toolbox for multiple sclerosis lesion segmentation. Neuroradiology 2015;57:1031-43 CrossRef Medline

23. Sweeney EM, Shinohara RT, Shiee N, et al. OASIS is Automated Statistical Inference for Segmentation, with applications to multiple sclerosis lesion segmentation in MRI. Neuroimage Clin 2013;2:402-13 CrossRef Medline
24. Lecler A, El Sanharawi I, El Methni J, et al. Improving detection of multiple sclerosis lesions in the posterior fossa using an optimized 3D-FLAIR sequence at 3T. AJNR Am J Neuroradiol 2019;40:1170-76 CrossRef Medline

25. Wang KY, Uribe TA, Lincoln CM. Comparing lesion detection of infratentorial multiple sclerosis lesions between T2-weighted spin-echo, 2D-FLAIR, and 3D-FLAIR sequences. Clin imaging 2018;51:229-34 CrossRef Medline

26. Prakkamakul S, Witzel T, Huang S, et al. Ultrafast brain MRI: clinical deployment and comparison to conventional brain MRI at 3T. J Neuroimaging 2016;26:503-10 CrossRef Medline

27. Longo MG, Conklin J, Cauley SF, et al. Evaluation of ultrafast waveCAIPI MPRAGE for visual grading and automated measurement of brain tissue volume. AJNR Am J Neuroradiol 2020;41:1388-96 CrossRef Medline

28. Sati P, Oh J, Constable RT, et al. NAIMS Cooperative. The central vein sign and its clinical evaluation for the diagnosis of multiple sclerosis: a consensus statement from the North American Imaging in Multiple Sclerosis Cooperative. Nat Rev Neurol 2016;12:714-22 CrossRef Medline

29. Absinta M, Sati P, Fechner A, et al. Identification of chronic active multiple sclerosis lesions on 3T MRI. AJNR Am J Neuroradiol 2018;39:1233-38 CrossRef Medline

30. Clarke MA, Pareto D, Pessini-Ferreira L, et al. Value of 3T susceptibility-weighted imaging in the diagnosis of multiple sclerosis. AJNR Am J Neuroradiol 2020;41:1001-08 CrossRef Medline

31. Absinta M, Sati P, Masuzzo F, et al. Association of chronic active multiple sclerosis lesions with disability in vivo. JAMA Neurol 2019;76:1474-83 CrossRef Medline 